\title{
Benefits of an open access echocardiography service: a Dutch prospective cohort study
}

\author{
N. van Gurp • L. J. M. Boonman-De Winter • \\ D. W. Meijer Timmerman Thijssen • \\ H. E. J. H. Stoffers
}

Published online: 23 May 2013

(C) The Author(s) 2013. This article is published with open access at Springerlink.com

\begin{abstract}
Background Open access echocardiography has been evaluated in the United Kingdom, but hardly in the Netherlands. The echocardiography service of the SHL-Groep in Etten-Leur was set up independently from the regional hospitals. Cardiologists not involved in the direct care of the participating patients evaluated the echocardiograms taken by ultrasound technicians. Aims We estimated the reduction in the number of referrals to regional cardiologists, the adherence of the general practitioners (GPs) to the advice of the evaluating cardiologist, GPs' opinion on the benefit of the echocardiography service and GPs' adherence to the diagnostic protocol advocated in the Dutch clinical guideline for heart failure.

Methods A prospective cohort study was performed. Patients were included from April 2011 to April 2012 ( $N=$ $155)$. Data from application forms $(N=155)$, echocardiography results $(N=155)$ and telephone interviews with GPs $(N$ $=138$ ) were analysed.

Results GPs referred less patients to the cardiologist than they would have done without echocardiography available ( $92 \%$ vs. $34 \%, p<0.001)$. They treated more patients by themselves (62\% vs. $10 \%, p<0.001)$. Most GPs $(81 \%$ ) followed the
\end{abstract}

The questions can be answered after the article has been published in print. You have to $\log$ in to: www.cvoi.nl.

N. van Gurp $(\bowtie) \cdot$ H. E. J. H. Stoffers

Department of General Practice/Family Medicine,

Maastricht University Medical Centre, School for Public Health and Primary Care (CAPHRI), P.O. Box 616, 6200 MD Maastricht, the Netherlands

e-mail: nicolevangurp@gmail.com

L. J. M. Boonman-De Winter

Scientific Research, SHL-groep, Trivium 76,

4873 LP Etten-Leur, the Netherlands

D. W. Meijer Timmerman Thijssen

SHL-groep, Trivium 76,

4873 LP Etten-Leur, the Netherlands advice presented on the echocardiogram result. Most GPs ( $82 \%$ ) found the service had clinical benefit for the patient. Sixty two percent of echocardiography requests met the criteria of the Dutch clinical guideline for heart failure.

Conclusion Open access echocardiography saved referrals to the cardiology department, saved time, and enabled GPs to treat more patients by themselves. Adherence to diagnostic guidelines for heart failure was suboptimal.

Keywords Echocardiography · General practice · Heart failure $\cdot$ Heart valve diseases $\cdot$ Prospective studies . Cohort studies

\section{Introduction}

Open access echocardiography is a diagnostic service for general practitioners (GPs) which enables them to obtain an echocardiogram for patients with suspected heart failure or valve disease, without referral to a cardiologist. In the United Kingdom (UK) most GPs have had access to this service for many years $[1,2]$. However, it is less available in the Netherlands. Often referral to a cardiologist is necessary for an echocardiogram and thus for diagnosing several cardiac conditions. Many of these referrals are unnecessary because not all patients have a cardiac condition and if they do, GPs are often able to manage them according to existing guidelines [3]. By providing GPs with access to echocardiography they can diagnose and treat several cardiac conditions by themselves.

Open access echocardiography has been evaluated in eastern South Limburg [4-7] and Zwolle [8]. To our knowledge, ours is the first Dutch study evaluating an echocardiography service, set up independently of the regional hospitals. External cardiologists not involved in the direct care of the participating patients evaluated the echocardiograms taken by ultrasound technicians. We investigated 
whether open access echocardiography would result in less referrals to cardiologists. Additionally, we assessed whether GPs adhered to the evaluating cardiologist's advice provided on the result form, and we asked GPs if they thought the service benefitted the patient. Furthermore, we investigated the adherence of GPs to the diagnostic protocol advocated by the Dutch clinical guideline for heart failure [3].

\section{Methods}

\section{Study design and population}

We performed a prospective cohort study in the southwest of the Netherlands. The echocardiography service was made available by SHL-Groep in Etten-Leur, a diagnostic centre which provides support services to primary care. Information on the service and an invitation to join a symposium in which the service was presented was sent to 316 GPs from 181 family practices. GPs were informed of the application procedure and restrictions for referral; patients younger than 18 years and patients with suspected acute cardiac conditions were not allowed to participate.

Patients referred to SHL-Groep for echocardiography between April 2011 and April 2012 were eligible. The Medical Ethics Committee of the Maastricht University Medical Centre approved the study.

\section{Measurements and variables}

We provided GPs with standardised request forms with tick boxes for the indication, relevant medical history, signs and symptoms, and results of diagnostic tests, i.e. electrocardiogram (ECG), N-terminal pro-brain natriuretic peptide (NTproBNP), and chest X-ray. On the application form we asked what the GP would have done with this patient if open access echocardiography had not been available. In case of incomplete forms, we contacted the GP by telephone before echocardiography results were known.

The telephonists of the SHL-Groep filled out a list every Monday, describing the waiting time for an appointment for echocardiography at that time.

Echocardiography was performed every Thursday by one of two ultrasound technicians using a Philips CX50 device. The images were parasternal long axis (PLAX), parasternal short axis (PSAX), and apical two, three, four, and five chamber views $(2 \mathrm{CH}, 3 \mathrm{CH}, 4 \mathrm{CH}, 5 \mathrm{CH})$. The inferior vena cava (IVC) was visualised subcostally. The technician posted the echocardiogram in a portal on a secured website.

One of two participating cardiologists from Erasmus Medical Centre evaluated the images within three working days. The cardiologists had access to the indication and patient reported length and weight. They classified systolic, diastolic and valve function according to the criteria of the American Society of Echocardiography and the European Association of Echocardiography [9-11]. Systolic function was determined with eyeballing, which was less time consuming than calculating ejection fraction [12]. Diastolic function was measured using mitral inflow (E/A ratio), tissue Doppler imaging of the mitral annulus (E/E' ratio), left atrial diameter, and left ventricle wall thickness. Colour flow Doppler was used to visualise the flow through the valves; the severity of valve disease was determined by eyeballing. The sniff test was used to measure IVC collapse. The echocardiography results with the conclusion and advice of the evaluating cardiologist were sent to the GP.

After the GP had received the results and contacted the patient, we asked in a telephone interview what management was initiated. We also inquired whether the GP had followed the evaluating cardiologist's advice, and whether they thought the echocardiogram had been of benefit. Finally, we asked GPs to estimate the waiting time for receiving an echocardiogram if patients would have been referred to a regional cardiologist.

\section{Data analysis}

Sample size To significantly demonstrate the expected difference between hypothetical referral to a regional cardiologist and actual referral after echocardiography, 26 patients were required. We calculated the sample size for McNemar's testing. We aimed at a power of 0.90 and a level of statistical significance of 0.05 for double-sided testing $(\alpha=0.05)$. We expected a 0.75 probability of referral before echocardiography, and a 0.25 probability of referral after echocardiography. Since patients were not burdened because of our study, we included more patients for the descriptive analyses.

Statistical analysis We used SPSS version 19.0 for Windows. Missing values were assumed to be missing completely at random. We checked outliers with the original data and corrected them if they proved to be wrongly copied. We used descriptive statistics to calculate frequencies. Confidence intervals are shown for continuous variables. With McNemar's test for discrete paired variables we investigated whether GPs would refer less to cardiologists with open access echocardiography available and manage more patients by themselves than they otherwise would have done.

Referring more than one patient per GP could cause a bias ('nesting') because patient management after echocardiography is GP-dependent. We assessed nesting by including only the first referred patient of each GP and compared this with the analysis including all eligible patients.

To calculate the GP participation rate the denominator was determined as all family practices within the range of $38 \mathrm{~km}$ from the diagnostic centre $(N=172)$. This was the distance to the remotest referring practice 'as the crow flies'. 
This was done because not every family practice informed of the existence of the service $(N=181)$ could reasonably be expected to refer to the echocardiography service because of the long distance.

\section{Results}

\section{Study population}

Participation Sixty-nine GPs from 54 practices referred at least one patient for an echocardiogram. Four GPs, together referring 17 patients, did not participate in the telephone interview.

Patients A total of 164 patients were referred to the open access service; no requests were refused. Informed consent was obtained from 156 patients (95\%). One patient was not eligible because he was under-age. Data of 155 patients were available for descriptive statistics. Table 1 shows the patient characteristics and Fig. 1 shows the indications.

In the analysis we used data of 105 patients with a complete application form and telephone interview (Fig. 2). The median time in days between performance of the echocardiogram and the interview was 133 (range 11-340) days.

Echocardiograms Eight of the 155 echocardiograms (5\%) were without any abnormality. Of the 80 patients referred for 'suspected valve disease' 34 (43\%) actually had valve disease, one result was missing. Of the 45 patients referred for 'suspected heart failure' 29 (64\%) actually had left ventricle dysfunction, ten results were missing. Missing results were the consequence of a temporary technical problem, which caused the result sheet to be incomplete.

Main outcome: referral to the cardiologist

In 97 of the 105 cases (92\%) the GP had indicated on the application form that he would have referred this patient to a regional cardiologist without echocardiography available. After the echocardiography results were known, only 36 patients $(34 \%)$ were actually referred $(p<0.001)$. The effect of 'nesting' on the results was checked by an analysis of a subset of patients including only the first patient per GP $(N=$ 44). The results were similar: $40(91 \%)$ versus 16 (36\%) patients, respectively $(p<0.001)$.

Beforehand no GP would have referred any patient to a specialist other than the cardiologist. After the echocardiogram $3(3 \%)$ patients were referred to another specialist, twice to the pulmonologist, once to the physiotherapist.

Finally, without the availability of open access echocardiography GPs intended to treat ten patients $(10 \%)$ by themselves. Having access to echocardiography, they treated 65 patients $(62 \%)$ themselves $(p<0.001)$.
Adherence to the evaluating cardiologist's advice

GPs were responsible for the management of the patients. Of all echocardiograms $(N=155), 31$ contained a specific advice from the evaluating cardiologist, consisting mostly of recommendations to refer to a regional cardiologist. GPs followed this suggestion in 25 patients $(81 \%)$. In one case the advice was not followed. Of five patients the actual management was unknown.

GPs' opinion on benefit of the echocardiography service

The GPs estimated the waiting time for a regular echocardiogram via a regional cardiologist at roughly 5 weeks. The average waiting time for an echocardiogram at the SHLGroep was 6 days.

In 127 cases (82\%) GPs thought the echocardiogram was of benefit for decision making, in 11 cases GPs thought it had no surplus value, and 17 results were missing.

GPs' adherence to the Dutch clinical guideline for heart failure

The guideline states that in case of suspected heart failure an ECG should be performed and (NT-pro)BNP should be determined; a chest X-ray is optional [3]. If the result of one of the first two tests is abnormal an echocardiogram is advised. Table 1 shows the diagnostic testing before referral to the open access echocardiography service. Of the 55 patients with suspected heart failure, 30 patients $(55 \%)$ had an NT-proBNP determined and in 34 cases $(62 \%)$ an ECG was performed. A chest X-ray was performed for 18 patients (33\%). For 34 patients (62\%) GPs had the results of the ECG and/or NT-proBNP, of which at least one was abnormal. For 13 patients (24\%) neither ECG nor NTproBNP was performed. Missing values were interpreted as not performed.

\section{Discussion}

Main findings

Our study shows that open access echocardiography may lead to significantly less referrals to the cardiologist (34\% vs. $92 \%, p<0.001)$. GPs were able to manage more patients in primary care $(p<0.001)$ and in most patients (82\%) GPs found the echocardiogram of benefit for decision making. In 25/31 cases GPs followed the evaluating cardiologist's advice. In this study, $62 \%$ of the 55 patients with suspected heart failure had either an abnormal ECG or NT-proBNP, whereas in $24 \%$ neither tests had been performed. 
Table 1 Patient characteristics of the population referred for open access echocardiography, as reported by their GPs on the application form $(N=155)$
${ }^{\text {a }}$ Positive family history for a cardiac disorder, sports-related indications, atrial fibrillation, or assessment of left ventricle hypertrophy

${ }^{\mathrm{b}}$ Diabetes, atrial fibrillation, cerebrovascular accidents, or COPD

${ }^{\mathrm{c}}$ Palpitations, chest pain, vertigo, fatigue, collapse, and cough

${ }^{\mathrm{d}}$ Blood pressure measurements, irregular heartbeat

${ }^{\mathrm{e}} \mathrm{C} / \mathrm{T}$ ratio: cardiothoracic ratio

\begin{tabular}{|c|c|c|}
\hline & Number of patients $(N=155)$ & Percentage \\
\hline Male & 59 & $38 \%$ \\
\hline Mean age in years $(95 \% \mathrm{CI})$ & $61.3(58.6-63.9)$ & - \\
\hline Mean body mass index in $\mathrm{kg} / \mathrm{m}^{2}(95 \% \mathrm{CI})$ & $27.8(27.0-28.6)$ & - \\
\hline \multicolumn{3}{|l|}{ Indication } \\
\hline - Suspected heart failure & 55 & $35 \%$ \\
\hline - Suspected valve disease & 81 & $52 \%$ \\
\hline - Other ${ }^{\mathrm{a}}$ & 37 & $24 \%$ \\
\hline - Missing & 1 & $1 \%$ \\
\hline \multicolumn{3}{|l|}{ Medical history } \\
\hline - Hypertension & 68 & $44 \%$ \\
\hline - Angina pectoris & 10 & $6 \%$ \\
\hline - Acute coronary syndrome & 3 & $2 \%$ \\
\hline - Other ${ }^{\mathrm{b}}$ & 31 & $20 \%$ \\
\hline - Missing & 5 & $3 \%$ \\
\hline \multicolumn{3}{|l|}{ Symptoms } \\
\hline - Reduced exercise capacity & 59 & $38 \%$ \\
\hline - Dyspnoea & 37 & $24 \%$ \\
\hline - Paroxysmal nocturnal dyspnoea and/or orthopnoea & 5 & $3 \%$ \\
\hline - Other ${ }^{\mathrm{c}}$ & 53 & $34 \%$ \\
\hline - Missing & 5 & $3 \%$ \\
\hline \multicolumn{3}{|l|}{ Physical examination } \\
\hline - Murmur & 72 & $46 \%$ \\
\hline - Oedema & 22 & $14 \%$ \\
\hline - Crepitation & 8 & $5 \%$ \\
\hline - Elevated jugular venous pressure & 5 & $3 \%$ \\
\hline - Third heart sound & 2 & $1 \%$ \\
\hline - Apex beat outside mid-clavicular line or heaving & 2 & $1 \%$ \\
\hline - Other ${ }^{\mathrm{d}}$ & 11 & $7 \%$ \\
\hline - Missing & 5 & $3 \%$ \\
\hline \multicolumn{3}{|l|}{ NT-proBNP } \\
\hline - Normal & 9 & $6 \%$ \\
\hline - Elevated & 28 & $18 \%$ \\
\hline - Not performed & 110 & $71 \%$ \\
\hline - Missing & 8 & $5 \%$ \\
\hline \multicolumn{3}{|l|}{ ECG } \\
\hline - Normal & 33 & $21 \%$ \\
\hline - Abnormal & 40 & $26 \%$ \\
\hline - Not performed & 75 & $48 \%$ \\
\hline - Missing & 7 & $5 \%$ \\
\hline \multicolumn{3}{|l|}{ X-thorax } \\
\hline - Normal C/T ratio & 25 & $16 \%$ \\
\hline$-\mathrm{C} / \mathrm{T}$ ratio $>0.5$ & 8 & $5 \%$ \\
\hline - Not performed & 116 & $75 \%$ \\
\hline - Missing & 6 & $4 \%$ \\
\hline
\end{tabular}

Strengths and limitations

Our study is the first prospective cohort study evaluating GP management following open access echocardiography.
Participation of GPs in the echocardiography service was low. This in contrast to the study participation, which was high for both patients (156/164 informed consent) and GPs (138/155 telephone interviews). Unfortunately, only 105 


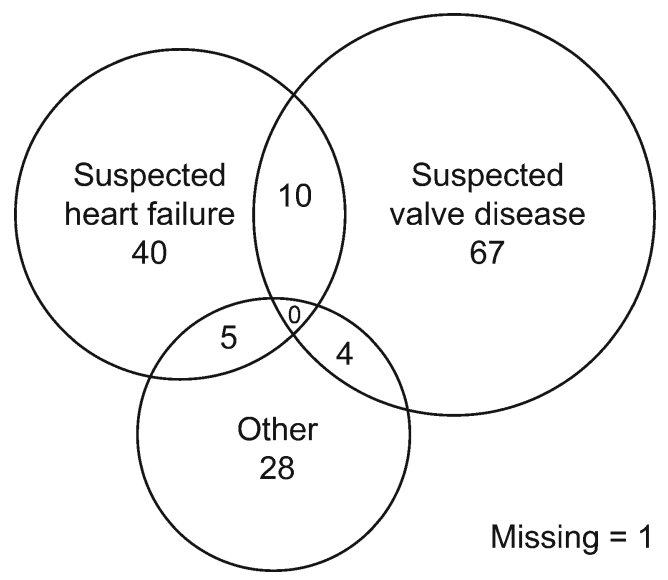

Fig. 1 Indication for referral to the open access echocardiography service to the SHL-groep $(N=155)$. * Numbers shown are numbers of patients

echocardiograms were available for the main analysis, mainly due to incomplete request forms. When starting the service, the quality of echocardiograms was not optimal according to the assessing cardiologists, presumably because of a lack of routine and experience of the ultrasound technicians. Despite of the suboptimal quality, the cardiologist was able to evaluate each echocardiogram. In case of doubt, he advised to refer the patient. The evaluating cardiologists provided feedback when necessary and the quality improved in the course of time.

At first the evaluating cardiologists did not structurally add an advice to the echocardiography results, only in case of pathology which required referral. Because other studies have suggested that GPs strongly appreciate the specialist's advice, the cardiologist was asked to provide an advice with every echocardiogram [13-15]. This change was later optimised by adding tick boxes for conclusion and advice in the reporting portal which generates the result sheet, both required to be filled in by the cardiologist to be able to proceed.

The time between performance of the echocardiogram and the telephone interview with the GPs had a wide range. To avoid a response bias (GPs may not accurately remember precise patient management initiated months ago), each GP was asked to check the patient's medical record during the telephone interview, assuming the record was accurate. The average follow-up time is short, which means there are no data on delayed referrals to the cardiologist, nor on hospitalisation for cardiac causes in patients who were initially not referred.

Analysing only one patient per GP instead of all patients did not change the results. Consequently, 'nesting' was thought not to be of influence.

Interpretation

Our study confirms the findings of Dutch and British studies that have suggested that open access echocardiography would lead to less 'unnecessary' referrals to the cardiologist $[8,15]$. The great majority of the evaluating cardiologists' advice ( $81 \%$ ) was followed by the GPs in our study. Van Heur et al. found that $71 \%$ of the cardiologist's advice to refer the patient was followed by the GP [4]. Francis et al. showed that open access echocardiography led to advice to change management in more than two-thirds of the patients [1]. However, whether the GPs in their study actually adhered to the advice was unknown.

British and Irish studies have reported that GPs think the option of performing an echocardiogram without referring the patient to the cardiologist is useful $[14,16,17]$. These
Fig. 2 Inclusion of patients for the study. * Numbers shown are numbers of patients

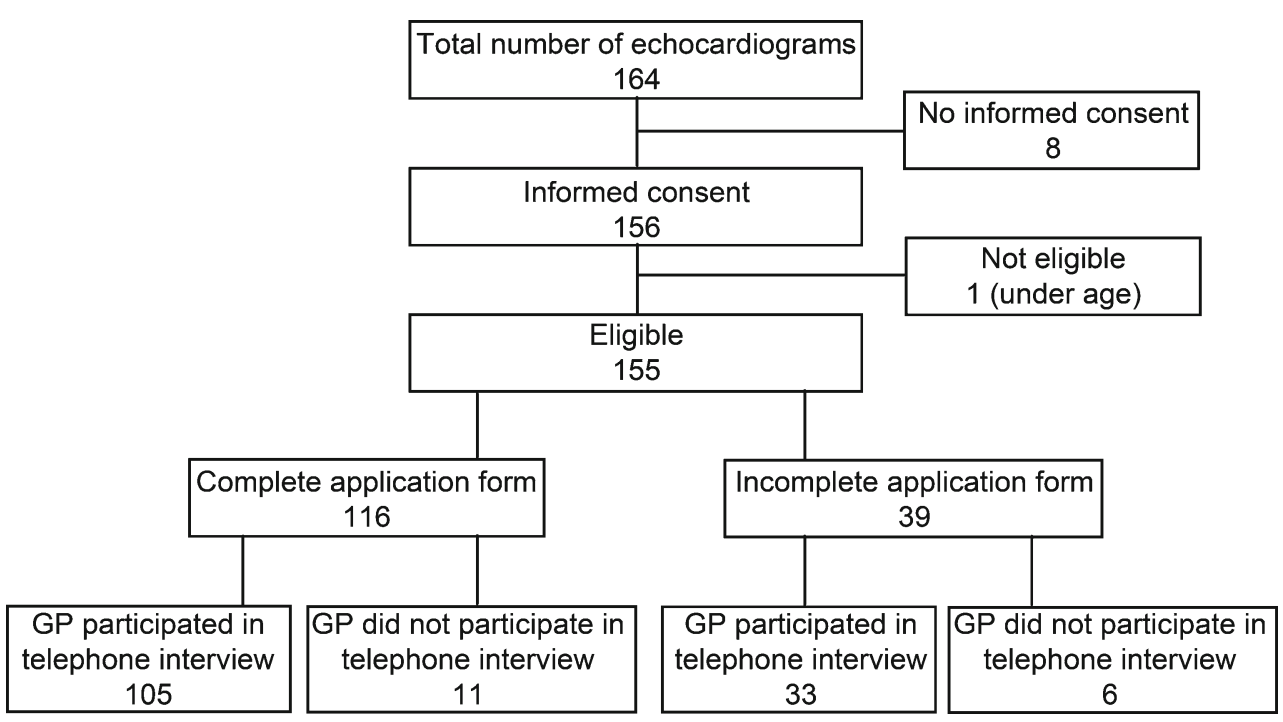


findings are confirmed by our study. The most probable reason for this opinion is the swiftness of the service (within a week). In a study from the UK, Williams et al. also found that waiting time for open access echocardiography is shorter than for the outpatient clinic [15].

The Dutch clinical guideline for heart failure advocates the use of ECG and NT-proBNP before performing echocardiography [3]. In our study, $24 \%$ of the patients referred for echocardiography did not have any of these tests, indicating room for improvement.

\section{Implications for practice}

Based on the results of our study and others, we think open access echocardiography could be very useful. Improvement of GP adherence to the diagnostic workup as advocated in the Dutch guideline on heart failure could make the echocardiography service more efficient. More research is needed to investigate its effect on the quality of care.

\section{Conclusion}

Open access echocardiography can have an important impact on patient management. The service saves referrals to the cardiology department, saves time, and enables GPs to treat more patients by themselves.

Acknowledgments We thank the GPs who participated in this study. Furthermore, we wish to thank C. Marien and L. van Meir (technicians, SHL-Groep) for performing the echocardiograms and we thank T.W. Galema MD PhD and M. L. Geleijnse MD PhD (cardiologists, Erasmus Medical Centre) for evaluating the echocardiograms. We are grateful for the statistical advice of B. Winkens $\mathrm{PhD}$ (statistician, Maastricht University Medical Centre).

\section{Declarations}

1. Ethical approval: the Medical Ethics Committee of the Maastricht University Medical Centre approved the study. The investigation conforms with the principles outlined in the Declaration of Helsinki.

2. Conflict of interest: Dirk W. Meijer Timmerman Thijssen and Leandra J.M. Boonman - De Winter are workers at the SHL-groep.

3. Funding: None.

Open Access This article is distributed under the terms of the Creative Commons Attribution License which permits any use, distribution, and reproduction in any medium, provided the original author(s) and the source are credited.

\section{References}

1. Francis C, Caruana L, Kearney P, et al. Open access echocardiography in management of heart failure in the community. BMJ. 1995;310(6980):634-6.

2. Colquhoun $M$, Waine $C$, Monaghan $M$, et al. Investigation in general practice of patients with suspected heart failure. How should the essential echocardiographic service be delivered? $\mathrm{Br}$ Heart J. 1995;74(4):335-6.

3. Hoes A, Voors A, Rutten F, et al. NHG-Standaard Hartfalen (Tweede herziening). Huisarts Wet. 2010;53(7):368-89.

4. van Heur L, Baur L, Tent M, et al. Evaluation of an open access echocardiography service in the Netherlands: a mixed methods study of indications, outcomes, patient management and trends. BMC Health Serv Res. 2010;10(1):37-46.

5. Baur L, Lenderink T, Lodewijks C, et al. Easy access echocardiography for the general practicioner: results from the Parkstad area in the Netherlands. Int J Cardiovasc Imaging. 2006;22(1):19-25.

6. Lodewijks-van der Bolt C, Baur L, Lenderink T, et al. The Dutch experience of open access echocardiography. Neth Heart J. 2007;15(10):342-7.

7. Baur L, Veenstra L, Lenderink T, et al. Open access echocardiography is feasible in the Netherlands. Neth Heart J. 2006;14(11):361-5.

8. Kluiver EP. Effecten van vrije toegang van de huisarts tot nietinvasief cardiaal functie-onderzoek: een nieuwe selectiemethode als alternatief voor directe verwijzing naar de cardiologische polikliniek. Rotterdam: Erasmus University Rotterdam; 2003.

9. Lang RM, Bierig M, Devereux RB, et al. Recommendations for chamber quantification: a report from the American Society of Echocardiography's Guidelines and Standards Committee and the Chamber Quantification Writing Group, developed in conjunction with the European Association of Echocardiography, a branch of the European Society of Cardiology. J Am Soc Echocardiogr. 2005;18(12):1440-63.

10. Khouri SJ, Maly GT, Suh DD, et al. A practical approach to the echocardiographic evaluation of diastolic function. J Am Soc Echocardiogr. 2004;17(3):290-7.

11. Grayburn M, Kraft CD, Nihoyannopoulos P, et al. Recommendations for evaluation of the severity of native valvular regurgitation with two-dimensional and Doppler echocardiography. J Am Soc Echocardiogr. 2003;16(7):777-802.

12. Sim V. Review finds inadequate evidence of the cost effectiveness and efficacy of open access echocardiography in primary care. Evid Based Cardiovasc Med. 2004;8(2):180-2.

13. Fuat A, Hungin APS, Murphy JJ. Barriers to accurate diagnosis and effective management of heart failure in primary care: qualitative study. BMJ. 2003;326(7382):196-201.

14. Mahadevan V, Earley M, McClements B. Open access echocardiography has diagnostic yield similar to outpatient echocardiography and is highly rated by general practitioners and patients. Int $\mathrm{J}$ Cardiol. 2005;99(3):389-93.

15. Williams S, Currie P, Silas J. Open access echocardiography: a prospective audit of referral patterns from primary care. Int J Clin Pract. 2003;57(2):136-9.

16. Horne R, Coombes I, Davies G, et al. Barriers to optimum management of heart failure by general practitioners. Br J Gen Pract. 1999;49(442):353.

17. Khunti K. Systematic review of open access echocardiography for primary care. Eur J Heart Fail. 2004;6(1):79. 


CVOI E-learning formula!
This is the CVOI e-learning article. The author has prepared 10 questions which are
available through the website of the Cardiovascular Educational Institute (CVOI).
Please follow the instructions below.
After finishing the questions you will be asked to fill in your name, hospital and e-mail address; then press the button
verzenden'.
When 6 out of the 10 questions are answered correctly, you acquire 1 accreditation point granted by the Quality Committee
of the Netherlands Society of Cardiology (NVVC). The acquired point will be credited to your personal file in the GAIA
system. You will also receive an e-mail with all the correct answers.
Over a period of one year 10 e-learning articles will appear in 10 subsequent NHJ editions. In each edition the e-learning
article will be recognisable by a special icon. On an annual basis you can collect 10 accreditation points. The accreditation
points are credited in the GAIA system by the CVOI.
If you need additional information, please contact the CVOI by e-mail: cvoi@cvoi.org or by phone: 030-2345001.
E.E. van der Wall
Chief editor NHJ
K.B. Schick
Coordinator CVOI

